\title{
Potential role of radiation therapy in augmenting the activity of immunotherapy for gynecologic cancers
}

\author{
Christina H Son' \\ Gini F Fleming ${ }^{2}$ \\ John W Moroney ${ }^{3}$ \\ 'Department of Radiation \& Cellular \\ Oncology, University of Chicago \\ Medicine, ${ }^{2}$ Section of Hematology/ \\ Oncology, Department of Medicine, \\ University of Chicago Medicine, \\ ${ }^{3}$ Section of Gynecologic Oncology, \\ Department of Obstetrics \& \\ Gynecology, University of Chicago \\ Medicine, Chicago, IL, USA
}

This article was published in the following Dove Press journal:

Cancer Management and Research

31 October 2017

Number of times this article has been viewed

\begin{abstract}
Immune checkpoint inhibitors have become an area of intense interest in oncology and are actively being studied in a variety of cancer types with a wide range of success. In vitro data suggest mechanisms by which radiation can activate the immune system, and ongoing studies are exploring the potential interaction of checkpoint inhibitors with radiotherapy in both preclinical and clinical settings. Gynecologic malignancies are a heterogeneous group of tumors with varying prognoses, intrinsic immunogenicity, and potential for response to immune-based therapies. In this review, we focus on the rationale for immunotherapy and opportunities for augmentation by photon radiotherapy in cancers of the cervix, endometrium, and ovary.
\end{abstract}

Keywords: immunotherapy, gynecologic, PD-1, radiation

\section{The rise of immunotherapy}

Based on our current understanding of tumor immunology, effective anti-tumor immunity can be achieved via 1) enhanced presentation of dendritic cells (DCs), 2) the promotion of productive T-cell responses, and 3) overcoming immunosuppressive signaling by malignant cells in the tumor microenvironment (TME) ${ }^{1}$ (Figure 1). Immunosuppression within the TME is most commonly a result of checkpoint inhibition mediated by numerous different effector cytokines, among which cytotoxic T-lymphocyte-associated protein 4 (CTLA-4) and programmed-death receptor 1 (PD-1) ligands are the best described. Antagonism of checkpoint inhibition is the area that has seen the most effective clinical advances within the past several years and will be discussed in this paper.

The recent advent of immune checkpoint inhibitors has been the source of renewed hope for patients with multiple malignancies, including those with historically poor outcomes, such as metastatic melanoma and lung cancer. Targeting CTLA-4 as well as PD-1 in melanoma has produced overall survival (OS) improvements in both the metastatic and adjuvant settings. ${ }^{2-4}$ Initial studies demonstrated that the CTLA-4 inhibitor ipilimumab (Bristol-Myers Squibb, New York City, NY, USA) (with or without a peptide vaccine) improved OS compared with a peptide vaccine alone in patients with previously treated advanced melanoma. ${ }^{5}$ Patients with ipilimumab-refractory disease were then found to have a significantly improved 6-month progression-free survival (PFS) of $34 \%-38 \%$ vs $16 \%$ with the use of PD-1 inhibition (via pembrolizumab; Merck \& Co., Kenilworth, NJ, USA) vs investigator-choice chemotherapy. ${ }^{6}$ Subsequently, a randomized comparison of pembrolizumab vs ipilimumab revealed the superiority of PD-1 inhibition, with a 6-month PFS of $46 \%$ vs $26.5 \%$ ( $p<0.001)$, respectively. ${ }^{4}$ This was followed by a Phase III study of the combination of a different PD-1 inhibitor (nivolumab; Bristol-Myers Squibb, New
Correspondence: Christina H Son Department of Radiation \& Cellular Oncology, University of Chicago, 5758 S. Maryland Ave. MC 9006, Chicago, IL 60637, USA

Tel +l $773795557 \mid$

Fax +I 7738347233

Email cson@radonc.uchicago.edu 


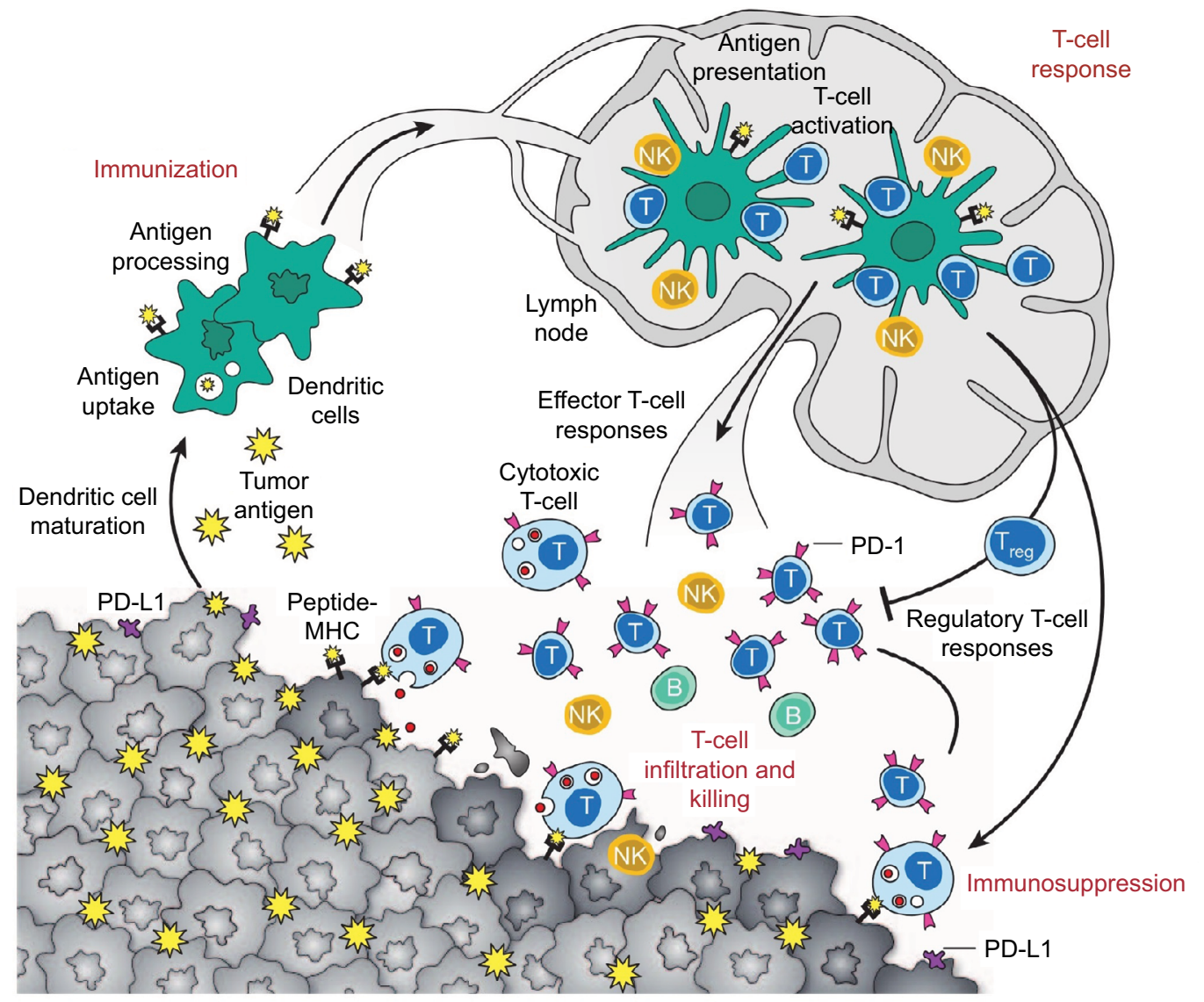

Figure I Anti-tumor immune response.'

Notes: DCs process and present tumor antigens on MHC proteins. In the presence of a maturation stimulus, DCs can trigger effector or regulatory T-cell responses. Effector T-cell responses lead to a protective effect and result in tumor cell killing, while regulatory T-cell responses lead to release of $\mathrm{T}_{\text {reg }}$ (regulatory $\mathrm{T}$-cells) and subsequent immunosuppressive effect mediated via various mechanisms, including PD-I and its ligand PD-LI. Reprinted by permission from Macmillan Publishers Ltd: Nature. Mellman I, Coukos G, Dranoff G. Cancer immunotherapy comes of age. Nature. 201 I;480(7378):480-489, copyright 201।.'

Abbreviations: DCs, dendritic cells; MHC, major histocompatibility complex; PD-I, programmed death receptor-I; PD-LI, programmed death ligand-I.

York City, NY, USA) plus ipilimumab vs ipilimumab alone vs nivolumab alone. Combination therapy resulted in improved PFS compared with either monotherapy option, but was also associated with higher rates of grade $3-4$ toxicity (55\%), mainly diarrhea, colitis, and increased transaminitis. ${ }^{7}$ These and similar successes have led to the investigation and US Food and Drug Administration approval of immune checkpoint inhibitors in multiple, disparate malignancies, including renal cell carcinoma, lung cancer, bladder cancer, Hodgkin lymphoma, and head and neck cancers. However, there are as yet no approved indications for gynecologic malignancies. Gynecologic cancers are a diverse group, ranging from virus-mediated cervical squamous cell carcinomas (SCCs) to hormone-modulated endometrial adenocarcinomas. Use of radiation therapy (RT) has the potential to augment the activity of immunotherapy in these cancers.

\section{Immune checkpoint inhibitors: mechanism of action}

Molecules that inhibit T-cell activation, such as CTLA-4, function to dampen the immune response and promote immune tolerance. CTLA-4 (expressed on helper CD4+ T-cells) binds to its ligand, B7 (found on antigen-presenting cells), acting as a co-inhibitory signal. Similarly, PD-1 is primarily expressed on effector T-cells and acts to inhibit the immune response. Its ligands, PD-L1 and PD-L2, are differentially expressed on various tissues: PD-L1 is presented on antigen-presenting cells, as well as non-hematopoietic cells, while PD-L2 is expressed on DC and macrophages. Both have been found to be expressed in many tumor cells, acting as the foundation for the development of anti-PD-1 and anti-PD-L1 therapies. ${ }^{8}$

\section{Radiation therapy and immunotherapy}

While response rates to checkpoint inhibitors have been impressive in malignancies such as melanoma and Hodgkin lymphoma, gynecologic cancers have, unfortunately, demonstrated significantly poorer response rates. ${ }^{9,10}$ Numerous correlative studies have established that some tumors are phenotypically "cold" or "T-cell non-inflamed" and less likely to respond to checkpoint inhibition than "hot" or 
"T-cell inflamed" tumors. ${ }^{11}$ Specifically, tumors with a T-cellinflamed microenvironment are characterized by infiltration of CD8+ T-cells, chemokines (such as CCL2, CCL3, CCL4, CCL5, CXCL9, and CXCL10) and an interferon signature. ${ }^{12}$

The ability of a tumor to induce an effective immune response is referred to as "immunogenicity," and is known to impact responsiveness to immunotherapeutic agents. Tumors that are felt to be highly immunogenic include melanoma and cancers of the bladder, lung, and kidney, with response rates of $20 \%-30 \%$ to $\mathrm{PD}-1$ inhibitors in the setting of advanced (and often pre-treated) disease. ${ }^{13-17}$ These response rates demonstrate that, even in the most promising tumor histologies, the majority of cancer patients do not respond to immunotherapy. This has led to interest in incorporating other therapeutic modalities to supplement and improve the immune response. One such option is RT. Historically, the mechanism of cell death after RT has been considered to be via deoxyribonucleic acid (DNA) damage, but it is now recognized that a component of tumor damage may be due to immunogenic cell death. ${ }^{18}$ Radiation causes release of tumor antigens, as well as the release of cytokines and chemokines, and increases expression of death receptors and other molecules that contribute to recognition of tumor cells by the immune system. ${ }^{19,20}$ The combination of radiation with immunotherapy has garnered significant interest due to the hypothesis that radiation can trigger tumor antigen release and enhance T-cell responses while concurrent or sequenced immunotherapeutics (such as targeted agents of PD-1 or CTLA-4) may work synergistically to suppress activity of regulatory T-cells (Figure 2).

\section{Preclinical investigations}

In preclinical studies, the addition of concurrent PD-L1 targeting to radiation was shown by Deng et al to improve both irradiated and distant tumor control of implanted breast and colorectal carcinomas in mice compared with single-modality therapy (PD-L1 or irradiation). ${ }^{21} \mathrm{~A}$ recently published study from the University of Pennsylvania using a mouse model of melanoma reported unique mechanisms by which antiCTLA-4, PD-L1 antibody, and RT work toward promoting the immune response against tumor cells. ${ }^{22}$ Radiation was noted to increase and diversify the intra-tumoral T-cell repertoire, while the CTLA-4 antibody was shown to enhance proliferation of T-cells and PD-L1 blockade enhanced tumorinfiltrating lymphocytes (TILs).

\section{Early clinical experience}

The majority of the available literature combining immunotherapy and radiotherapy involves the use of ipilimumab, a

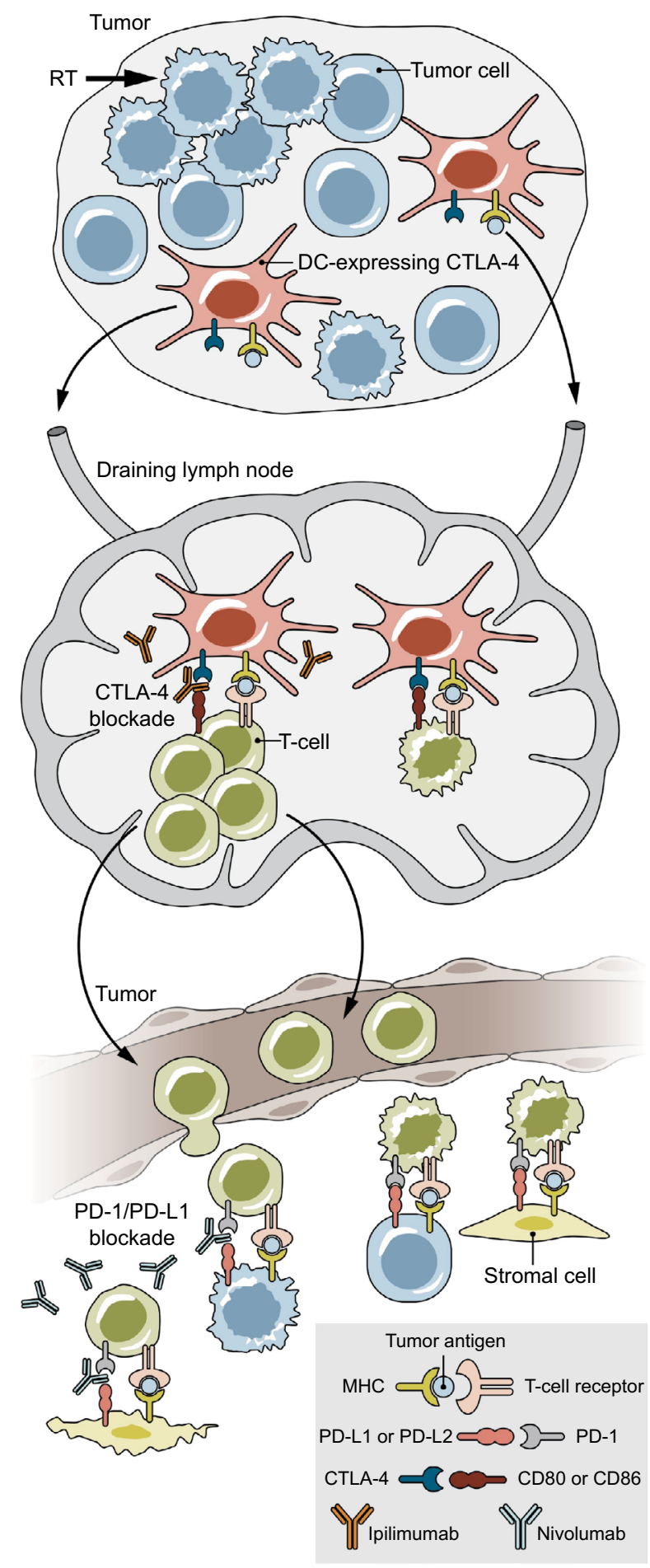

Figure 2 The role of radiotherapy and checkpoint inhibition on immune targeting of tumor cells.

Notes: Radiation enhances antigen presentation on DCs, which travel to the draining lymph node and can activate T-cell expansion. Effector T-cells in the tumor microenvironment are then able to target tumor and stromal cells for killing. However, CTLA-4 on DCs inhibits T-cell expansion and PD-I on effector cells (including regulatory T-cells) causes T-cell apoptosis, leading to an immunesuppressive response. Thus, blockade of CTLA-4 and PD-I (or its ligand PD-LI or PDL2, expressed on cancer and other cells in the tumor microenvironment) can enhance an immune response. From Spiotto M, Fu YX, Weichselbaum RR. The intersection of radiotherapy and immunotherapy: mechanisms and clinical implications. Sci Immunol. 2016; I (3):EAAG I 266. Reprinted with permission from AAAS..$^{20}$

Abbreviations: CTLA-4, cytotoxic T-lymphocyte-associated protein 4; DCs, dendritic cells; PD-I, programmed death receptor-I; PD-LI, programmed death ligand-I; RT, radiation therapy. 
CTLA-4 antibody. Case reports have suggested a possible abscopal effect in patients receiving RT and ipilumimab. ${ }^{23,24}$ The abscopal effect occurs when there is regression of other, non-irradiated sites of disease after RT. These case reports demonstrate near-complete radiographic responses with sustained tumor control. . $^{2324}$

The ideal fractionation and dose of radiation to obtain the most immunogenic response has not been established, and a preclinical study in mouse models of breast and colon carcinoma suggests greater tumor responses (both in-field and out of field) with 3-5 fractions (total dose 24-30 Gy) rather than a single fraction $(20 \mathrm{~Gy})$ in the setting of antiCTLA-4 monoclonal antibody. ${ }^{25}$ A separate study of radiation alone revealed greater tumor-specific T-cell responses (splenic interferon-gamma [IFN- $\gamma]$ ) when higher radiation doses (5-15 Gy in a single fraction) were administered. ${ }^{26}$ However, the proportion of CD4+/CD25+/Foxp3+ $\mathrm{T}_{\text {reg }}$ cells also varied with radiation dose. At a dose of $5 \mathrm{~Gy}$, there was no increased IFN- $\gamma$ response. At the dose of 15 Gy, there was a large increase in effector T-cell response, but this was also accompanied by the greatest increase in proportion of $\mathrm{T}_{\text {reg }}$ cells. The investigators thus stated that the greatest benefit (increase in tumor-specific IFN- $\gamma$ with decrease in $\mathrm{T}_{\text {reg }}$ cells) appeared to be at doses of 7.5 and $10 \mathrm{~Gy}$, although only the 7.5 Gy dose demonstrated statistical significance in proportion of $\mathrm{T}_{\text {reg }}$ cells compared with no radiation. When the investigators studied the effect of fractionation, the greatest response observed was when the total dose of 15 Gy was divided into 2 fractions, rather than 3 or 5 fractions. ${ }^{26}$

In clinical practice, large doses of radiation (typically $\geq 5$ Gy per fraction) delivered over 5 or fewer fractions (often referred to as stereotactic body RT, or SBRT) have been used to ablate tumors in those patients where local control of the tumor is felt to be important. This is used in both the definitive (e.g., inoperable lung cancer) and metastatic settings. While the tolerability and efficacy of the combination of radiation and an anti-PD-1/PD-L1 checkpoint inhibitor has not yet been prospectively demonstrated in the clinical setting, each individual modality is typically associated with low rates of severe toxicity. Studies of PD-1 inhibitors alone report $<15 \%$ grade 3-4 toxicity for single-agent pembrolizumab in advanced melanoma ${ }^{4,27}$ and $<20 \%$ for nivolumab ${ }^{28,29}$ in metastatic renal cell carcinoma. SBRT alone has been shown to be very tolerable with $<15 \%$ grade $3-4$ toxicity using a 3-5 fraction regimen to total doses of 40-60 Gy. ${ }^{30-32}$

Numerous studies that combine SBRT to various body sites and tumor types with immune checkpoint inhibitor therapy are ongoing or completed. KEYNOTE-001 was a
Phase I study of pembrolizumab in patients with advanced or metastatic non-small cell lung cancer. A secondary analysis of 98 patients enrolled on the KEYNOTE-001 trial from a single institution was performed to determine if the use of previous RT had an effect on disease outcome. ${ }^{33}$ Patients who had received prior RT had a longer time from initial diagnosis compared with those who had not received RT, but were otherwise well-matched. Nearly $30 \%$ of patients in the RT group had received SBRT or stereotactic radiosurgery. RT was associated with improved overall and PFS, even after accounting for other factors such as age, performance status, smoking status, sex, or histology. Although there was higher treatment-related pulmonary toxicity in the RT group $(13 \%$ vs $1 \%, p<0.05)$, there was no difference in grade 3 or higher pulmonary toxicity.

In prostate cancer, 2 large randomized studies combining RT with ipilimumab have been published. ${ }^{34,35}$ The first, a multi-institutional, Phase III, randomized controlled trial included nearly 800 men with docetaxel-refractory metastatic castrate-resistant prostate cancer. ${ }^{34} \mathrm{All}$ men received a single $8 \mathrm{~Gy}$ fraction of radiation to a bone metastasis followed by either ipilimumab ( $10 \mathrm{mg} / \mathrm{kg}$ every 3 weeks for up to 4 doses) or placebo within 2 days of irradiation. Although there was no difference in the primary endpoint of OS, the hazard ratio decreased over time, such that ipilimumab was associated with improved survival at longer follow-up time points. Additionally, a PFS benefit was noted. Toxicity, however, was notably increased in the ipilumumab arm (26\% grade $3-4$ immune-related toxicity rate vs $3 \%$ in the placebo group). The authors concluded that there is sufficient activity with ipilimumab in prostate cancer to warrant further study. While the benefit of RT was unclear in this particular trial (due to study design), it is unclear, as noted previously, whether a single fraction of 8 Gy would be sufficient to achieve the desired immunogenic effect. A Phase I/II study explored the use of escalating doses of ipilimumab $(3-10 \mathrm{mg} / \mathrm{kg})$ in men with metastatic castrate-resistant prostate cancer; some patients also received an 8 Gy single-fraction radiation dose to bone metastases within 24-48 hours of drug delivery. ${ }^{35}$ Investigators noted that $\sim 15 \%$ of patients experienced a decline in PSA $\geq 50 \%$ in the group receiving $10 \mathrm{mg} / \mathrm{kg}$ of drug; no statistical comparison was made between the patients who received RT vs no RT. Although the maximum tolerated dose was not reached, the rate of any grade 3-4-related toxicity was 38\% in the highest dose group $(10 \mathrm{mg} / \mathrm{kg})$ receiving radiation.

Another group reported on 22 patients with metastatic melanoma who received 2-3 fractions of 6-8 Gy per fraction to a single lesion followed within 3-5 days by 4 cycles of 
ipilimumab (3 mg/kg). The majority of patients $(64 \%)$ had progressive disease in the un-irradiated lesions on this regimen; there were no dose-limiting toxicities (defined as any grade 4 immune-related toxicity or any grade 3 immune-related toxicity within 30 days after the last ipilimumab dose). ${ }^{22}$

\section{Cervical cancer}

Cervical cancer rates in the USA have decreased considerably in recent decades, with $\sim 12,990$ estimated new cases diagnosed in 2016 , making it $<1 \%$ of all new cancer cases ${ }^{36}$ However, the global burden of disease remains large. Recent data from the World Health Organization International Agency for Research on Cancer suggest that it is the fourth most common cancer in women worldwide, with 528,000 new cases in 2012, and developing countries accounting for $\sim 85 \%$ of these cases.

The majority of cervical cancers are a result of persistent infection with high-risk subtypes of human papillomavirus (HPV). Subsequent expression of viral oncogenes, E6 and E7, which interact with p53 and retinoblastoma tumor suppressor gene proteins, respectively, results in dysregulated cell cycle control and apoptosis. ${ }^{37}$ Management of early-stage cervical cancers usually involves regional therapy such as radical hysterectomy or pelvic RT. Early-stage patients are generally treated with surgery and have a very good chance of cure, with 5-year relative survival of $>90 \%$ for "localized" disease. ${ }^{36}$ Those with large tumors $(>4 \mathrm{~cm})$ or clinically apparent extension beyond the cervix (parametria or pelvic lymph nodes) have a lower likelihood of cure with survival rates of $60 \%-70 \% .{ }^{36}$ These patients are generally treated with concurrent chemoradiation (concomitant cisplatin) followed by a boost to the primary tumor using intracavitary or interstitial brachytherapy techniques. Those with the highest risk of disease include women with extensive lymphatic spread to the para-aortic or supraclavicular regions; these patients have dismal survival rates of $30 \%-60 \% .{ }^{38}$ Reports of patterns of failure suggest that distant recurrences are the primary component of failures, but locoregional failures can also occur. $^{38,39}$ The OUTBACK trial (ANZGOG 0902/GOG 0274/ RTOG 1174, NCT01414608) is a randomized controlled trial studying the addition of adjuvant carboplatin and paclitaxel following chemoradiation for patients with locally advanced cervical cancer, with an OS endpoint.

Because of the viral pathogenesis of cervical cancer, and the demonstrated success of prophylactic vaccines that prevent cervical dysplasia and potentially invasive disease, there has been sustained interest in the use of immunotherapy agents for treatment of affected patients. There are currently numerous ongoing trials studying the safety and efficacy of therapeutic vaccines; all of these vaccines target the HPV proteins E6 and/or E7. The DNA vaccine VGX-3100 has already demonstrated some success in improving regression rates of CIN-2 and CIN-3. ${ }^{40}$ Engineered autologous T-cells with chimeric antigen receptors are also being studied as a means of boosting tumor-specific immune response. ${ }^{41}$ Although vaccine technology has improved over time, most trials with therapeutic vaccines have thus far met with limited success. ${ }^{42}$

\section{Somatic immune mutational status and response to therapy}

Hodgkin lymphoma patients have been noted to have very high $(>80 \%)$ response rates to the PD-1 inhibitor nivolumab, and in an analysis of those with available tissue, all of the responders were noted to have copy number gain of 2 particular genes that encode PD-L1 and PD-L2 ${ }^{43}$ Two-thirds of cervical SCC have been noted to have mutations in these same genes: co-amplification or co-gain (as determined by FISH) of CD274 (encodes PD-L1) and PCD1LG2 (encodes PD-L2) at 9p24.1 was associated with increased expression of PD-L1. In a small group of 23 vulvar cancer patients that were also included in this study, presence of the mutation appeared to be independent of p16 status. ${ }^{44}$

\section{Tumor immune-microenvironment}

A report by Heeren et al compared lymph nodes involved with cervical cancer with uninvolved lymph nodes. They found that involved lymph nodes had fewer CD4+ and more CD8+ T-cells, as well as increased surface levels of PD-1 and CLTA-4 on lymphocytes. Additionally, there were increased levels of regulatory cells (regulatory antigen-presenting cells, FoxP3+ regulatory T-cells, and other suppressor cells). ${ }^{45} \mathrm{~A}$ follow-up report by the same group suggests that the immune microenvironment is similar (elevated regulatory T-cells) in the lymph node basins that are anatomically closer to the tumorcontaining lymph node compared with those that are anatomically distant from the involved lymph node. ${ }^{46}$ This is similar to a finding in HPV-associated head and neck SCC, in which investigators noted that expression of PD-L1 was increased at the site of deep tonsillar crypts, the site at which initial infection is felt to occur. However, PD-L1 expression was not found at the surface epithelium, leading the investigators to suggest that the deep crypts may represent a distinctive immune-privileged site and a mechanism by which HPV-associated head and neck cancers may evade detection. In this small series, $70 \%$ of HPV-associated tumors expressed PD-L1, while only $29 \%$ of non-HPV-associated tumors expressed PD-L1 ${ }^{47}$ 


\section{Therapeutic modalities in cervical cancer}

Potential mechanisms to overcome an immune-suppressive microenvironment include augmentation of checkpoint inhibitor therapy with chemotherapy and/or radiotherapy. Cisplatin, the mainstay of chemotherapy in cervical cancer, may act to modulate myeloid cells in the tumor TME to improve the immune response to tumor cells; additionally, in the setting of vaccines, it has been shown to act synergistically with vaccine-induced T-cells to promote tumor cell death. ${ }^{48,49}$

The KEYNOTE-028 study of pembrolizumab in advanced cervical SCC with PD-L1 expression in $\geq 1 \%$ of tumor or stroma cells (by immunohistochemistry [IHC]) revealed an objective response rate of $12.5 \% .{ }^{9}$ Unfortunately, while there is general consensus that PD-L1-positive tumors respond better to immunotherapy agents than biomarker negative tumors, there is significant variation in both assay thresholds and techniques that makes the use of PD-L1 by IHC a predictive factor for response to PD-1 and PD-L1 inhibitors problematic. ${ }^{50}$ Two trials currently sponsored by the National Cancer Institute include patients with recurrent or metastatic cervical cancer. NCT02257528 is a Phase II study of nivolumab, while NCT01693783 is a Phase II study of ipilimumab. Early results from the latter study have been presented, demonstrating partial response in 3 of 34 patients. ${ }^{51}$ Ipilimumab is also being studied in a Phase I safety study as an adjuvant therapy after chemoradiation in the upfront setting for locally advanced cervical cancer patients (NCT01711515).

Given the low response rate observed in KEYNOTE-028 with pembrolizumab alone, there may be potential augmentation of immune response with the addition of radiotherapy via the mechanisms noted above. These opportunities exist in both the upfront and metastatic settings. As discussed previously, ongoing early-phase studies of radiotherapy and checkpoint inhibitors typically utilize ablative radiation doses via SBRT. Brachytherapy is another form of dose escalation; however, it can differ from SBRT in many ways (including dose rate, homogeneity of dose distribution over the target area, and varying number of fraction sizes) that could lead to differences in the radiobiologic effect in tissues. In locally advanced cervical cancer, it is typically delivered through intracavitary (via the vaginal canal) or interstitial (via needles placed through the perineum) methods as a standard component of definitive chemoradiation. Varying fractionation schemes can be considered for high-dose rate brachytherapy per the American Brachytherapy Society guidelines, including 4-6 fractions of 5-7 Gy per fraction. ${ }^{52}$
Preclinical data specifically studying brachytherapy and the immune environment are scarce, but in one report, investigators using transgenic mice found that after administration of a vaccine against the carcinoembryonic antigen, the addition of external beam radiation or brachytherapy induced CD8+ T-cell responses in the spleen that were specific for tumor-associated antigens not encoded by the vaccine..$^{53}$ Brachytherapy, in this study, consisted of an Iodine-125 (I-125) seed at a dose rate of $4 \mathrm{cGy}$ per hour. This is a much lower dose rate than modern high-dose rate sources, which are in excess of $1200 \mathrm{cGy}$ per hour. Low-dose rate sources also remain in use, with typical rates of $<200 \mathrm{cGy}$ per hour. Similarly, preliminary results of a study from the University of Iowa reported on mice inoculated with lymphoma cells. One group received an injection of a toll-like receptor 9 (TLR9) agonist (vs no intervention); another group received brachytherapy (using I-125) with or without the TLR9 agonist. Tumor-specific CD8 response in the spleen was highest in the group receiving the TLR9 agonist with brachytherapy ${ }^{54}$ Both of these studies demonstrated the ability of RT to augment tumor-specific immune responses.

In a more recent prostate cancer study, serum samples from men receiving a variety of cancer therapies was collected. The proportion of patients developing antibody responses to tumor proteins was noted to be $29 \%$ in those receiving hormone therapy, $14 \%$ in those undergoing external beam radiation, and $25 \%$ after brachytherapy. No patients undergoing radical prostatectomy developed an immune response. ${ }^{55}$ Although there are very few data specifically studying brachytherapy and the immune environment, the available preclinical studies, including extrapolation from SBRT (high doses delivered via external beam RT rather than with brachytherapy), suggest a rationale for further investigation of brachytherapy in combination with immunotherapeutic agents.

\section{Endometrial cancer}

Uterine cancers are the most common gynecologic cancer in the USA, with an estimated 60,050 new cases in 2016. Standard of care for these patients included surgical resection with or without adjuvant chemotherapy or radiation. Cancers confined to the uterus (stage I) are generally treated with either observation or radiotherapy following surgery based on clinical and pathologic risk features. Adjuvant radiotherapy can take the form of either brachytherapy targeted to the vaginal apex or external beam radiotherapy targeted to the pelvis (or both). Generally, stage I patients 
have a very good prognosis. Aggressive histologic subtypes (including grade 3 endometrioid-type adenocarcinoma, clear cell, papillary serous, or carcinosarcoma) are often treated with chemotherapy even in the stage I setting, although we lack prospective data showing that this improves outcomes. Locoregionally advanced cancers are often treated with a combination of both chemotherapy and radiotherapy in an attempt to control both distant and locoregional recurrences. Serous or carcinosarcoma subtypes have a much more dismal prognosis, with large population-based studies suggesting 5 -year survival rates of $<50 \% .^{56-58}$

Analysis of endometrioid and serous histologic subtypes of endometrial cancer (those included in the cancer genome atlas study) confirmed 4 molecular subtypes: POLE mutated, microsatellite instability (MSI) hypermutated, copy-number low, and copy-number high. ${ }^{59}$ POLE is a component of the DNA polymerase epsilon, and endometrial cancer patients with this mutation ( $\sim 10 \%$ of patients) have the best prognosis of the 4 subtypes, with PFS of nearly $100 \%$, even in grade 3 tumors. ${ }^{59,60}$ MSI hypermutated and copy-number low tumors have an intermediate prognosis, while copy-number high tumors (including the vast majority of serous tumors) have the worst prognosis and commonly are associated with mutations in TP53. Additionally, molecular analysis demonstrated that approximately one-quarter of high-grade endometrioid adenocarcinomas shared similar molecular characteristics with serous carcinomas (mostly in the copynumber high group). Approximately $40 \%$ of endometrioid tumors and $2 \%$ of serous tumors were noted to be MSIhypermutated. ${ }^{59}$ This can be a result of a germline mutation in DNA mismatch repair genes (hereditary nonpolyposis colorectal cancer, or Lynch syndrome) or due to sporadic mismatch repair-deficient tumors due to somatic mutations or epigenetic silencing. ${ }^{61}$

Both MSI- and POLE-mutated tumors have been noted to have features considered to be favorable for response to anti-PD-1 therapies, likely due to a high burden of mutations leading to greater production of neoantigens available for immune targeting. ${ }^{62,63}$ In a study of endometrial cancer patients, investigators have shown overexpression of PD-1 in the TILs and peritumoral lymphocytes of these tumors, as well as increased PD-L1 expression. Neoantigen load was highest in the POLE-mutated tumors, followed by the MSI tumors. It was lowest in the microsatellite stable (MSS) tumors. ${ }^{62}$ Clinical outcomes seem to corroborate the significance of these favorable features of the TME, with MSI-mutated tumors (including endometrial primary tumors) demonstrating very good response rates to the PD-1 inhibitor pembrolizumab. ${ }^{63}$ In those with MSI colorectal cancers, the objective response rate was $40 \%$, while in the non-colorectal cancer patients (including endometrial primary tumors), the objective response rate was $71 \%$. In those with MSS colorectal cancers, the objective response rate was $0 \%$. When comparing those with MSI vs MSS tumors in the colorectal cancer population, there was a statistically significant difference in OS after administration of the drug (not reached in the MSI group vs 5 months in the MSS group). Pembrolizumab has recently been approved by the FDA for advanced MSI tumors regardless of histologic origin.

Investigators have also demonstrated that $P O L E$-mutated tumors appear to be more immunogenic, with tumorspecific activation of CD4+ T-cells and naïve CD4+ and CD8+ T-cells. ${ }^{64}$ These findings, along with other evidence suggesting that these tumors have greater resistance to platinum chemotherapeutic agents ${ }^{65}$ imply that perhaps the improved prognosis associated with these tumors is due to their increased stimulation of the immune system. While clinical reports studying the responsiveness of these particular tumors to PD-1 inhibition have not yet been described in a large population of patients, case reports have been published suggesting that there may be excellent response in these patients. ${ }^{66,67}$

While response rates to checkpoint inhibition for patients with POLE- and MSI-mutated tumors are excellent, responses in unselected patients are quite low: preliminary results for pembrolizumab from KEYNOTE-028 in endometrial cancer patients revealed an overall response rate of $13 \% .{ }^{10}$ Recently reported preliminary data studying Atezolizumab (Roche, Switzerland) (a PD-L1 inhibitor) also noted a 13\% response rate with duration $>7$ months in the 2 patients with response. ${ }^{68}$ As previously noted, the proportion of patients with $P O L E$ - or MSI-mutated tumors is low to moderate (especially in the high-risk serous tumors). It is likely even lower in patients with metastatic or advanced disease given the good prognosis associated with these mutations (particularly POLE). As noted in the Cancer Genome Atlas (TCGA) analysis, 94\% of the serous histology tumors were in the copy-number high group - the group with the lowest mutational burden and worst prognosis. ${ }^{59}$ For those patients for whom somatic testing does not suggest sensitivity to PD-1 inhibitors alone, augmentation of the immune response with radiation could be considered in the form of stereotactic body radiotherapy in the metastatic setting, or brachytherapy or fractionated external beam radiation in the upfront setting.

Ongoing protocols that include endometrial patients are studying the use of PD-1 inhibitors in the neoadjuvant setting 
prior to surgery (NCT02630823, NCT02728830) or in the advanced or recurrent setting (NCT02549209).

\section{Ovarian cancer}

Ovarian cancer is the second most common gynecologic cancer in the USA, with an estimated 22,280 new cases diagnosed in 2016. Outcomes are poor in this disease, with 5 -year survival rates $<50 \% .{ }^{36}$ The majority of ovarian cancers are epithelial in origin (most commonly serous histology), with other histologic types including germ cell or sex cordstromal tumors. The current treatment paradigm for ovarian cancers includes surgical debulking followed by observation (if the tumor is low grade and early stage) or adjuvant chemotherapy, typically with a taxane and carboplatin. Unfortunately, median PFS for those with advanced, extrapelvic (stage III) disease after adjuvant therapy is approximately 2 years or less. ${ }^{69-71}$

Based on patterns of failure data suggesting that most initial relapses are abdominopelvic, consolidative radiation targeted to the whole abdomen has been studied. However, based on current guidelines, there is no role for radiotherapy in most patients with epithelial ovarian cancers. Older studies examining whole-abdomen irradiation in the adjuvant setting demonstrated inconsistent results regarding efficacy compared with chemotherapy and raised significant concerns regarding toxicity, especially as related to bone marrow suppression and bowel obstruction. ${ }^{72-75}$ However, other small studies suggest potential benefit with the addition of radiation, with the caveat that the chemotherapy regimens used are outdated..$^{76,77}$ Newer, more conformal radiation techniques (such as intensitymodulated RT, or IMRT), can improve coverage of peritoneal surfaces and reduce dose to organs-at-risk, including bone marrow. Results from a German study, including a small group of patients with optimally debulked stage III ovarian cancer demonstrated that whole-abdomen irradiation using IMRT (30 Gy in 1.5 Gy per fraction) was well tolerated after 6 cycles of adjuvant carboplatin and taxane-based chemotherapy, with no reported grade 4 toxicity or treatment breaks. ${ }^{78-80}$ Relapse-free survival was 27.6 months. However, the primary pattern of failure continued to be in the peritoneum following irradiation, suggesting that further improvements are required in adjuvant therapies for this patient population. ${ }^{78}$

Previously published studies have described the tumor immune microenvironment in ovarian cancer patients. Curiel et al described their findings that CD4+CD25+FOXP3+ regulatory T-cells in the tumor were associated with worse clinical outcomes. ${ }^{81}$ These $\mathrm{T}_{\text {reg }}$ cells also were noted to be in close physical proximity to CD8+ TILs and to suppress proliferation of CD3+CD25-T-cells, IFN-gamma, and interleukin-2. The presence of tumor $\mathrm{T}_{\text {reg }}$ cells was independently associated with worse survival after controlling for stage of disease and extent of surgical debulking. The investigators also noted a dose effect, with increasing numbers of $\mathrm{T}_{\text {reg }}$ cells present in tumor being associated with progressively worse outcomes. ${ }^{81}$ Another group of investigators noted that CD8+ TILs and high CD8+/ $T_{\text {reg }}$ ratios were associated with improved survival in ovarian cancer patients. ${ }^{82} \mathrm{~A}$ separate study suggested that the prognostic significance of CD8+ TILs was limited to those patients who had undergone a complete (R0) debulking procedure, rather than an "optimal" ( $<1 \mathrm{~cm}$ residual disease) or incomplete cytoreduction. ${ }^{83}$

Tumor genomic studies also reveal subtypes of ovarian cancer that may be more impacted by immune manipulation. Gene expression profiling has improved the definitions of the original 4 ovarian the TCGA subtypes and has been found to have prognostic significance, with the "immunoreactive-like" tumors associated with the best survival and the "proliferative-like" and "mesenchymal-like" tumors having the worst outcomes. ${ }^{84,85}$ The fourth type, "differentiated-like" has an intermediate prognosis. The "immunoreactive-like" tumors have high expression of genes encoding major histocompatibility complex I and II, which are components of antigen presentation. PD-L1 was also differentially expressed in this group, suggesting a mechanism for immune evasion..$^{85}$

The success of therapeutic vaccines in ovarian cancer has been limited but demonstrates the potential for modulating the immune system in achieving improved outcomes for this patient population. ${ }^{86}$ Checkpoint inhibitors have also been studied in ovarian cancer, with a CTLA-4 inhibitor being studied in the setting of vaccine therapy with granulocytemacrophage colony-stimulating factor modified irradiated autologous tumor cells (GVAX) ${ }^{87}$ This study demonstrated reasonable tolerability and at least stable disease in nearly half the patients. Investigators also found that tumor regression was proportional to the $\mathrm{CD} 8+/ \mathrm{T}_{\text {reg }}$ ratio, suggesting that response could potentially be improved through the deactivation or targeting of $\mathrm{T}_{\text {reg }}$ cells ${ }^{87}$ More recently, investigators have published an early experience with nivolumab in 20 women with platinum-resistant ovarian cancer with an overall response rate of $15 \%$ and disease control rate of $45 \%$. Two patients had a durable ( $>350$ days) complete response, while 1 had a partial response. Median survival was 20 months. ${ }^{88}$ Preliminary results from the KEYNOTE-028 study of pembrolizumab in 26 women with advanced ovarian epithelial, fallopian tube, or primary peritoneal carcinoma and PD-L1 expression were also reported. Overall response rate by 
Response Evaluation Criteria in Solid Tumors (RECIST) was $11.5 \%$, but $23 \%$ had "evidence of tumor reduction." 89 The PD-L1 inhibitor avelumab (Merck KGaA, Germany) has also been studied, with preliminary data indicating a response rate of $17.4 \% .{ }^{90}$

Radiation has been studied previously as a chemosensitizer in patients with recurrent ovarian cancer receiving docetaxel in a Phase I study by the Gynecologic Oncology Group. ${ }^{91}$ Thirteen patients received low doses of wholeabdomen radiation (60 cGy, twice a day for 2 days per week, continuing for 6 weeks) using anterior-posterior fields concurrently with 3 different dosing regimens of weekly docetaxel $\left(20,25\right.$, or $\left.30 \mathrm{mg} / \mathrm{m}^{2}\right)$. The regimen was considered to be tolerable at the $20 \mathrm{mg} / \mathrm{m}^{2}$ dose level, as only 1 out of 7 patients enrolled at this dose level experienced grade 4 toxicity. However, median PFS was just over 3 months and no objective responses were seen. Based on preclinical data, larger fraction sizes may improve the sensitization provided by radiation in the setting of checkpoint inhibitor therapies, but toxicity and tolerability concerns may limit this approach and should be further studied in select patients who are at highest risk of recurrence.

\section{Conclusion}

Early studies of immune checkpoint inhibitors in gynecologic malignancies show some promise but, with the exception of MSI tumors, response rates have been limited, and no immune checkpoint inhibitors are currently approved for treatment of gynecologic malignancies. Preclinical studies demonstrate the ability of RT to augment the immune response, and standard of care for many gynecologic malignancies (particularly cervical cancer and endometrial cancer) already incorporates radiation. Therefore, studying combinations of immunotherapy and RT to establish tolerability and evaluate efficacy in both the metastatic and curative setting is a logical next step. Additionally, further development and refinement of biomarkers to define patient selection criteria for both immunotherapy and radiotherapy is necessary to optimize the therapeutic ratio.

\section{Disclosure}

The authors report no conflicts of interest in this work.

\section{References}

1. Mellman I, Coukos G, Dranoff G. Cancer immunotherapy comes of age. Nature. 2011;480(7378):480-489.

2. Eggermont AM, Chiarion-Sileni V, Grob JJ, et al. Adjuvant ipilimumab versus placebo after complete resection of high-risk stage III melanoma (EORTC 18071): a randomised, double-blind, phase 3 trial. Lancet Oncol. 2015;16(5):522-530.
3. Robert C, Long GV, Brady B, et al. Nivolumab in previously untreated melanoma without BRAF mutation. $N$ Engl J Med. 2015;372(4): 320-330.

4. Robert C, Schachter J, Long GV, et al; KEYNOTE-006 investigators. Pembrolizumab versus ipilimumab in advanced melanoma. $N$ Engl $J$ Med. 2015;372(26):2521-2532.

5. Hodi FS, O'Day SJ, McDermott DF, et al. Improved survival with ipilimumab in patients with metastatic melanoma. $N$ Engl J Med. 2010;363(8):711-723.

6. Ribas A, Puzanov I, Dummer R, et al. Pembrolizumab versus investigator-choice chemotherapy for ipilimumab-refractory melanoma (KEYNOTE-002): a randomised, controlled, phase 2 trial. Lancet Oncol. 2015;16(8):908-918.

7. Larkin J, Chiarion-Sileni V, Gonzalez R, et al. Combined nivolumab and ipilimumab or monotherapy in untreated melanoma. N Engl J Med. 2015;373(1):23-34.

8. Boussiotis VA. Molecular and biochemical aspects of the PD-1 checkpoint pathway. $N$ Engl J Med. 2016;375(18):1767-1778.

9. Frenel JS, Tourneau CL, Neil BH, et al. Pembrolizumab in patients with advanced cervical squamous cell cancer: Preliminary results from the phase Ib KEYNOTE-028 study. J Clin Oncol. 2016;(Suppl 34):5515.

10. Ott PA, Bang YJ, Rigaud DB, et al. Pembrolizumab in advanced endometrial cancer: Preliminary results from the phase Ib KEYNOTE-028 study. J Clin Oncol. 2016;(Suppl 34):5581.

11. Gajewski TF. The next hurdle in cancer immunotherapy: overcoming the non-T-cell-inflamed tumor microenvironment. Semin Oncol. 2015;42(4):663-671.

12. Corrales L, Matson V, Flood B, Spranger S, Gajewski TF. Innate immune signaling and regulation in cancer immunotherapy. Cell Res. 2017;27(1):96-108.

13. Brahmer J, Reckamp KL, Baas P, et al. Nivolumab versus docetaxel in advanced squamous-cell non-small-cell lung cancer. $N$ Engl J Med. 2015;373(2):123-135.

14. Borghaei H, Paz-Ares L, Horn L, et al. Nivolumab versus docetaxel in advanced nonsquamous non-small-cell lung cancer. $N$ Engl J Med. 2015;373(17):1627-1639.

15. Plimack ER, Bellmunt J, Gupta S, et al. Safety and activity of pembrolizumab in patients with locally advanced or metastatic urothelial cancer (KEYNOTE-012): a non-randomised, open-label, phase 1b study. Lancet Oncol. 2017;18(2):212-220.

16. Motzer RJ, Escudier B, McDermott DF, et al. Nivolumab versus everolimus in advanced renal-cell carcinoma. $N$ Engl J Med. 2015;373(19): 1803-1813.

17. Weber JS, D'Angelo SP, Minor D, et al. Nivolumab versus chemotherapy in patients with advanced melanoma who progressed after anti-CTLA-4 treatment (CheckMate 037): a randomised, controlled, open-label, phase 3 trial. Lancet Oncol. 2015;16(4):375-384.

18. Golden EB, Frances D, Pellicciotta I, Demaria S, Helen Barcellos-Hoff M, Formenti SC. Radiation fosters dose-dependent and chemotherapyinduced immunogenic cell death. Oncoimmunology. 2014;3:e28518.

19. Formenti SC, Demaria S. Combining radiotherapy and cancer immunotherapy: a paradigm shift. J Natl Cancer Inst. 2013;105(4):256-265.

20. Spiotto M, Fu YX, Weichselbaum RR. The intersection of radiotherapy and immunotherapy: mechanisms and clinical implications. Sci Immunol. 2016;1(3):EAAG1266.

21. Deng L, Liang H, Burnette B, et al. Irradiation and anti-PD-L1 treatment synergistically promote antitumor immunity in mice. J Clin Invest. 2014;124(2):687-695.

22. Twyman-Saint Victor C, Rech AJ, Maity A, et al. Radiation and dual checkpoint blockade activate non-redundant immune mechanisms in cancer. Nature. 2015;520(7547):373-377.

23. Postow MA, Callahan MK, Barker CA, et al. Immunologic correlates of the abscopal effect in a patient with melanoma. $N$ Engl J Med. 2012;366(10):925-931.

24. Golden EB, Demaria S, Schiff PB, Chachoua A, Formenti SC. An abscopal response to radiation and ipilimumab in a patient with metastatic non-small cell lung cancer. Cancer Immunol Res. 2013;1(6):365-372. 
25. Dewan MZ, Galloway AE, Kawashima N, et al. Fractionated but not single-dose radiotherapy induces an immune-mediated abscopal effect when combined with anti-CTLA-4 antibody. Clin Cancer Res. 2009;15(17):5379-5388.

26. Schaue D, Ratikan JA, Iwamoto KS, McBride WH. Maximizing tumor immunity with fractionated radiation. Int J Radiat Oncol Biol Phys. 2012;83(4):1306-1310.

27. Ribas A, Puzanov I, Dummer R, et al. Pembrolizumab versus investigator-choice chemotherapy for ipilimumab-refractory melanoma (KEYNOTE-002): a randomised, controlled, phase 2 trial. Lancet Oncol. 2015;16(8):908-918.

28. McDermott DF, Drake CG, Sznol M, et al. Survival, durable response, and long-term safety in patients with previously treated advanced renal cell carcinoma receiving nivolumab. J Clin Oncol. 2015;33(18):2013-2020.

29. Motzer RJ, Rini BI, McDermott DF, et al. Nivolumab for metastatic renal cell carcinoma: results of a randomized phase II trial. J Clin Oncol. 2015;33(13):1430-1437.

30. Ranck MC, Golden DW, Corbin KS, et al. Stereotactic body radiotherapy for the treatment of oligometastatic renal cell carcinoma. Am J Clin Oncol. 2013;36(6):589-595.

31. Salama JK, Hasselle MD, Chmura SJ, et al. Stereotactic body radiotherapy for multisite extracranial oligometastases: final report of a dose escalation trial in patients with 1 to 5 sites of metastatic disease. Cancer. 2012;118(11):2962-2970.

32. Stinauer MA, Kavanagh BD, Schefter TE, et al. Stereotactic body radiation therapy for melanoma and renal cell carcinoma: impact of single fraction equivalent dose on local control. Radiat Oncol. 2011;6:34.

33. Shaverdian N, Lisberg AE, Bornazyan K, et al. Previous radiotherapy and the clinical activity and toxicity of pembrolizumab in the treatment of non-small-cell lung cancer: a secondary analysis of the KEYNOTE-001 phase 1 trial. Lancet Oncol. 2017;18(7):895-903.

34. Kwon ED, Drake CG, Scher HI, et al; CA184-043 Investigators. Ipilimumab versus placebo after radiotherapy in patients with metastatic castration-resistant prostate cancer that had progressed after docetaxel chemotherapy (CA184-043): a multicentre, randomised, double-blind, phase 3 trial. Lancet Oncol. 2014;15(7):700-712.

35. Slovin SF, Higano CS, Hamid O, et al. Ipilimumab alone or in combination with radiotherapy in metastatic castration-resistant prostate cancer: results from an open-label, multicenter phase I/II study. Ann Oncol. 2013;24(7):1813-1821.

36. Howlader N NA, Krapcho M, Garshell J, et al editors. SEER Cancer Statistics Review. 1975-2013. National Cancer Institute: Bethesda, MD; 2016.

37. Munger K, Phelps WC, Bubb V, Howley PM, Schlegel R. The E6 and E7 genes of the human papillomavirus type 16 together are necessary and sufficient for transformation of primary human keratinocytes. $J$ Virol. 1989;63(10):4417-4421.

38. Kim JY, Kim JY, Kim JH, Yoon MS, Kim J, Kim YS. Curative chemoradiotherapy in patients with stage IVB cervical cancer presenting with paraortic and left supraclavicular lymph node metastases. Int J Radiat Oncol Biol Phys. 2012;84(3):741-747.

39. Beriwal S, Gan GN, Heron DE, et al. Early clinical outcome with concurrent chemotherapy and extended-field, intensity-modulated radiotherapy for cervical cancer. Int J Radiat Oncol Biol Phys. 2007;68(1): 166-171.

40. Trimble CL, Morrow MP, Kraynyak KA, et al. Safety, efficacy, and immunogenicity of VGX-3100, a therapeutic synthetic DNA vaccine targeting human papillomavirus 16 and 18 E6 and E7 proteins for cervical intraepithelial neoplasia 2/3: a randomised, double-blind, placebocontrolled phase 2b trial. Lancet. 2015;386(10008):2078-2088.

41. Eskander RN, Tewari KS. Immunotherapy: an evolving paradigm in the treatment of advanced cervical cancer. Clin Ther. 2015;37(1): 20-38.

42. van der Burg SH, Arens R, Ossendorp F, van Hall T, Melief CJ. Vaccines for established cancer: overcoming the challenges posed by immune evasion. Nat Rev Cancer. 2016;16(4):219-233.
43. Ansell SM, Lesokhin AM, Borrello I, et al. PD-1 blockade with nivolumab in relapsed or refractory Hodgkin's lymphoma. $N$ Engl $J$ Med. 2015;372(4):311-319.

44. Howitt BE, Sun HH, Roemer MG, et al. Genetic basis for PD-L1 expression in squamous cell carcinomas of the cervix and vulva. JAMA Oncol. 2016;2(4):518-522.

45. Heeren AM, Koster BD, Samuels S, et al. High and interrelated rates of PD-L1+CD14+ antigen-presenting cells and regulatory T cells mark the microenvironment of metastatic lymph nodes from patients with cervical cancer. Cancer Immunol Res. 2015;3(1):48-58.

46. Heeren AM, de Boer E, Bleeker MC, et al. Nodal metastasis in cervical cancer occurs in clearly delineated fields of immune suppression in the pelvic lymph catchment area. Oncotarget. 2015;6(32):32484-32493.

47. Lyford-Pike S, Peng S, Young GD, et al. Evidence for a role of the PD1:PD-L1 pathway in immune resistance of HPV-associated head and neck squamous cell carcinoma. Cancer Res. 2013;73(6):1733-1741.

48. Kang TH, Mao CP, Lee SY, et al. Chemotherapy acts as an adjuvant to convert the tumor microenvironment into a highly permissive state for vaccination-induced antitumor immunity. Cancer Res. 2013;73(8):2493-2504.

49. van der Sluis TC, van Duikeren S, Huppelschoten S, et al. Vaccineinduced tumor necrosis factor-producing $\mathrm{T}$ cells synergize with cisplatin to promote tumor cell death. Clin Cancer Res. 2015;21(4):781-794.

50. Khagi Y, Kurzrock R, Patel SP. Next generation predictive biomarkers for immune checkpoint inhibition. Cancer Metastasis Rev. 2017;36(1):179-190.

51. Lheureux S, Butler MO, Clarke B, et al. A phase I/II study of ipilimumab in women with metastatic or recurrence cervical carcinoma: A study of the Princess Margaret and Chicago N01 Consortia. J Clin Oncol. 2015;(33 Suppl):3061.

52. Viswanathan AN, Beriwal S, De Los Santos JF, et al; American Brachytherapy Society. American Brachytherapy Society consensus guidelines for locally advanced carcinoma of the cervix. Part II: high-dose-rate brachytherapy. Brachytherapy. 2012;11(1):47-52.

53. Hodge JW, Sharp HJ, Gameiro SR. Abscopal regression of antigen disparate tumors by antigen cascade after systemic tumor vaccination in combination with local tumor radiation. Cancer Biother Radiopharm. 2012;27(1):12-22.

54. Rich N, Dahle C, Modrick J, Buatti J, Weiner G. Brachytherapy combined with $\mathrm{CpG}$ ODN enhances development of a tumor antigen-specific CD8 response. Blood. 2004;104(11):4635.

55. Nesslinger NJ, Sahota RA, Stone B, et al. Standard treatments induce antigen-specific immune responses in prostate cancer. Clin Cancer Res. 2007;13(5):1493-1502.

56. del Carmen MG, Birrer M, Schorge JO. Uterine papillary serous cancer: a review of the literature. Gynecol Oncol. 2012;127(3):651-661.

57. Hamilton CA, Cheung MK, Osann K, et al. Uterine papillary serous and clear cell carcinomas predict for poorer survival compared to grade 3 endometrioid corpus cancers. Br J Cancer. 2006;94(5):642-646.

58. Nemani D, Mitra N, Guo M, Lin L. Assessing the effects of lymphadenectomy and radiation therapy in patients with uterine carcinosarcoma: a SEER analysis. Gynecol Oncol. 2008;111(1):82-88.

59. Cancer Genome Atlas Research Network, Kandoth C, Schultz N, et al. Integrated genomic characterization of endometrial carcinoma. Nature. 2013;497(7447):67-73.

60. Church DN, Stelloo E, Nout RA, et al. Prognostic significance of POLE proofreading mutations in endometrial cancer. J Natl Cancer Inst. 2015;107(1):402.

61. Herman JG, Umar A, Polyak K, et al. Incidence and functional consequences of hMLH1 promoter hypermethylation in colorectal carcinoma. Proc Natl Acad Sci U S A. 1998;95(12):6870-6875.

62. Howitt BE, Shukla SA, Sholl LM, et al. Association of polymerase e-mutated and microsatellite-instable endometrial cancers with neoantigen load, number of tumor-infiltrating lymphocytes, and expression of PD-1 and PD-L1. JAMA Oncol. 2015;1(9):1319-1323.

63. Le DT, Uram JN, Wang H, et al. PD-1 blockade in tumors with mismatch-repair deficiency. $N$ Engl J Med. 2015;372(26):2509-2520. 
64. Bellone S, Centritto F, Black J, et al. Polymerase epsilon (POLE) ultramutated tumors induce robust tumor-specific CD4+ T cell responses in endometrial cancer patients. Gynecol Oncol. 2015;138(1):11-17.

65. Bellone S, Bignotti E, Lonardi S, et al. Polymerase epsilon (POLE) ultra-mutation in uterine tumors correlates with $\mathrm{T}$ lymphocyte infiltration and increased resistance to platinum-based chemotherapy in vitro. Gynecol Oncol. 2017;144(1):146-152.

66. Mehnert JM, Panda A, Zhong H, et al. Immune activation and response to pembrolizumab in POLE-mutant endometrial cancer. J Clin Invest 2016;126(6):2334-2340.

67. Santin AD, Bellone S, Buza N, et al. Regression of chemotherapyresistant polymerase epsilon (POLE) ultra-mutated and MSH6 hyper-mutated endometrial tumors with nivolumab. Clin Cancer Res. 2016;22(23):5682-5687.

68. Fleming GF, Emens LA, Eder JP, et al. Clinical activity, safety and biomarker results from a phase Ia study of atezolizumab (atezo) in advanced/recurrent endometrial cancer (rEC). J Clin Oncol. 2017; 35(15)(Suppl):5585.

69. Chan JK, Brady MF, Penson RT, et al. Weekly vs. every-3-week paclitaxel and carboplatin for ovarian cancer. $N$ Engl J Med. 2016;374(8): 738-748.

70. Pignata S, Scambia G, Katsaros D, et al; Gynecologic Cancer Inter Group (GCIG) Investigators. Carboplatin plus paclitaxel once a week versus every 3 weeks in patients with advanced ovarian cancer (MITO-7): a randomised, multicentre, open-label, phase 3 trial. Lancet Oncol. 2014;15(4):396-405.

71. Katsumata N, Yasuda M, Isonishi S, et al; Japanese Gynecologic Oncology Group. Long-term results of dose-dense paclitaxel and carboplatin versus conventional paclitaxel and carboplatin for treatment of advanced epithelial ovarian, fallopian tube, or primary peritoneal cancer (JGOG 3016): a randomised, controlled, open-label trial. Lancet Oncol. 2013;14(10):1020-1026.

72. Redman CW, Mould J, Warwick J, et al. The west midlands epithelial ovarian cancer adjuvant therapy trial. Clin Oncol ( $R$ Coll Radiol). 1993;5(1):1-5.

73. Chiara S, Conte P, Franzone P, et al. High-risk early-stage ovarian cancer. Randomized clinical trial comparing cisplatin plus cyclophosphamide versus whole abdominal radiotherapy. Am J Clin Oncol. 1994;17(1):72-76.

74. Bruzzone M, Repetto L, Chiara S, et al. Chemotherapy versus radiotherapy in the management of ovarian cancer patients with pathological complete response or minimal residual disease at second look. Gynecol Oncol. 1990;38(3):392-395.

75. Lawton F, Luesley D, Blackledge G, et al. A randomized trial comparing whole abdominal radiotherapy with chemotherapy following cisplatinum cytoreduction in epithelial ovarian cancer. West Midlands Ovarian Cancer Group Trial II. Clin Oncol (R Coll Radiol). 1990;2(1):4-9.

76. Sorbe B, Swedish-Norgewian Ovarian Cancer Study G. Consolidation treatment of advanced (FIGO stage III) ovarian carcinoma in complete surgical remission after induction chemotherapy: a randomized, controlled, clinical trial comparing whole abdominal radiotherapy, chemotherapy, and no further treatment. Int J Gynecol Cancer. 2003;13(3):278-286
77. Einhorn N, Trope C, Ridderheim M, Boman K, Sorbe B, Cavallin-Stahl E. A systematic overview of radiation therapy effects in ovarian cancer. Acta Oncol. 2003;42(5-6):562-566.

78. Rochet N, Lindel K, Katayama S, et al. Intensity-modulated whole abdomen irradiation following adjuvant carboplatin/taxane chemotherapy for FIGO stage III ovarian cancer: four-year outcomes. Strahlenther Onkol. 2015;191(7):582-589.

79. Rochet N, Kieser M, Sterzing F, et al. Phase II study evaluating consolidation whole abdominal intensity-modulated radiotherapy (IMRT) in patients with advanced ovarian cancer stage FIGO III--the OVARIMRT-02 Study. BMC Cancer. 2011;11:41.

80. Rochet N, Sterzing F, Jensen AD, et al. Intensity-modulated whole abdominal radiotherapy after surgery and carboplatin/taxane chemotherapy for advanced ovarian cancer: phase I study. Int J Radiat Oncol Biol Phys. 2010;76(5):1382-1389.

81. Curiel TJ, Coukos G, Zou L, et al. Specific recruitment of regulatory $\mathrm{T}$ cells in ovarian carcinoma fosters immune privilege and predicts reduced survival. Nat Med. 2004;10(9):942-949.

82. Sato E, Olson SH, Ahn J, et al. Intraepithelial CD8+ tumor-infiltrating lymphocytes and a high $\mathrm{CD} 8+$ /regulatory $\mathrm{T}$ cell ratio are associated with favorable prognosis in ovarian cancer. Proc Natl Acad Sci USA. 2005;102(51):18538-18543.

83. Wouters MC, Komdeur FL, Workel HH, et al. Treatment regimen, surgical outcome, and T-cell differentiation influence prognostic benefit of tumor-infiltrating lymphocytes in high-grade serous ovarian cancer. Clin Cancer Res. 2016;22(3):714-724.

84. Cancer Genome Atlas Research Network. Integrated genomic analyses of ovarian carcinoma. Nature. 2011;474(7353):609-615.

85. Konecny GE, Wang C, Hamidi H, et al. Prognostic and therapeutic relevance of molecular subtypes in high-grade serous ovarian cancer. J Natl Cancer Inst. 2014;106(10):dju249.

86. Liu B, Nash J, Runowicz C, Swede H, Stevens R, Li Z. Ovarian cancer immunotherapy: opportunities, progresses and challenges. J Hematol Oncol. 2010;3:7.

87. Hodi FS, Butler M, Oble DA, et al. Immunologic and clinical effects of antibody blockade of cytotoxic T lymphocyte-associated antigen 4 in previously vaccinated cancer patients. Proc Natl Acad Sci US A. 2008;105(8):3005-3010.

88. Hamanishi J, Mandai M, Ikeda T, et al. Safety and antitumor activity of anti-PD-1 antibody, nivolumab, in patients with platinum-resistant ovarian cancer. J Clin Oncol. 2015;33(34):4015-4022.

89. Varga A, Piha-Paul SA, Ott PA, et al. Antitumor activity and safety of pembrolizumab in patients (pts) with PD-L1 positive advanced ovarian cancer: interim results from a phase Ib study. J Clin Oncol. 2015; 33(15)(Suppl):5510.

90. Disis ML, Patel MR, Pant S, et al. Avelumab (MSB0010718C), an anti-PD-L1 antibody, in patients with previously treated, recurrent or refractory ovarian cancer: A phase Ib, open-label expansion trial. J Clin Oncol. 2015;33(15)(Suppl):5509.

91. Kunos CA, Sill MW, Buekers TE, et al. Low-dose abdominal radiation as a docetaxel chemosensitizer for recurrent epithelial ovarian cancer: a Phase I study of the Gynecologic Oncology Group. Gynecol Oncol. 2011;120(2):224-228.
Cancer Management and Research

\section{Publish your work in this journal}

Cancer Management and Research is an international, peer-reviewed open access journal focusing on cancer research and the optimal use of preventative and integrated treatment interventions to achieve improved outcomes, enhanced survival and quality of life for the cancer patient The manuscript management system is completely online and includes

\section{Dovepress}

a very quick and fair peer-review system, which is all easy to use. Visit http://www.dovepress.com/testimonials.php to read real quotes from published authors. 